Research Article

\title{
Sex Variations in the Oral Microbiomes of Youths with Severe Periodontitis
}

\author{
Ya-Qiong Zhao, ${ }^{1}$ Ying-Hui Zhou, ${ }^{2}$ Jie Zhao, ${ }^{1}$ Yao Feng, ${ }^{1}$ Zheng-Rong Gao, ${ }^{1}$ Qin Ye, ${ }^{1}$ \\ Qiong Liu, ${ }^{1}$ Yun Chen, ${ }^{1}$ Shao-Hui Zhang, ${ }^{1}$ Li Tan, ${ }^{1}$ Marie Aimee Dusenge, ${ }^{1}$ Jing Hu, \\ Yun-Zhi Feng, ${ }^{1}$ Fei Yan $\mathbb{D}^{3},{ }^{3}$ and Yue Guo ${ }^{1}{ }^{1}$ \\ ${ }^{1}$ Department of Stomatology, The Second Xiangya Hospital of Central South University, Changsha 410011, Hunan, China \\ ${ }^{2}$ National Clinical Research Center for Metabolic Diseases, Hunan Provincial Key Laboratory of Metabolic Bone Diseases, \\ and Department of Metabolism and Endocrinology, The Second Xiangya Hospital of Central South University, Changsha 410011, \\ Hunan, China \\ ${ }^{3}$ Hunan Key Laboratory of Oral Health Research \& Hunan 3D Printing Engineering Research Center of Oral Care \& Hunan \\ Clinical Research Center of Oral Major Diseases and Oral Health \& Xiangya Stomatological Hospital \& Xiangya School \\ of Stomatology, Central South University, Changsha 410008, China
}

Correspondence should be addressed to Fei Yan; yfann2004@csu.edu.cn and Yue Guo; guoyue@csu.edu.cn

Received 2 June 2021; Revised 17 September 2021; Accepted 4 October 2021; Published 20 October 2021

Academic Editor: Jôice D. Corrêa

Copyright (c) 2021 Ya-Qiong Zhao et al. This is an open access article distributed under the Creative Commons Attribution License, which permits unrestricted use, distribution, and reproduction in any medium, provided the original work is properly cited.

\begin{abstract}
Objective. Periodontitis is an inflammatory disease of microbial etiology caused primarily by dysbiosis of the oral microbiota. Our aim was to compare variations in the composition of the oral microbiomes of youths with severe periodontitis according to gender. Methods. Subgingival plaque samples collected from 17 patients with severe periodontitis (11 males and 6 females) were split for $16 \mathrm{~S}$ rRNA gene sequencing. The composition, $\alpha$-diversity, and $\beta$-diversity of the patients' oral microbiomes were compared between the males and the females. Linear discriminant analysis effect size (LEfSe) was used to analyze the specific taxa enriched in the two groups. Functional profiles (KEGG pathways) were obtained using PICRUSt based on 16S rRNA gene sequencing data. Results. The Chaol index and phylogenetic diversity whole tree were significantly higher in males than in females. The Simpson and Shannon indices were not significantly different between the two groups. $\beta$-Diversity suggested that the samples were reasonably divided into groups. The Kruskal-Wallis test based on the relative abundance of species, combined with the LEfSe analysis showed that the dominant bacteria in males were Pseudomonas and Papillibacter, whereas the dominant bacteria in women were Fusobacteriales and Tannerella. KEGG analysis predicted that the variation in the oral microbiome may be related to the immune system in women, whereas immune system diseases were the dominant pathway in men. Conclusion. We found sex-specific differences in the oral microbiome in a sample of youths with severe periodontitis. The differences may be related to changes in immune homeostasis and lead to a better understanding of periodontitis.
\end{abstract}

\section{Introduction}

Disturbances in the oral microbiota can cause an immune response by the host that affects the protection and support of the periodontium, resulting in the development of periodontal disease [1]. Periodontitis is a highly prevalent oral disease among adults, with a prevalence of up to $50 \%$ in developed countries [2]. The prevalence is even greater $(\sim 90 \%)$ in developing countries [3]. The global burden of periodontitis is increasing with life expectancy and due to a worldwide decrease in tooth loss. In the 4th National Oral Health Survey in mainland China, the frequency of adults with periodontitis was $52.8 \%$ and with severe periodontitis (stage III or IV) $10.6 \%$ [4]. Understanding the composition and structure of oral microbiomes could improve periodontitis prevention, making it important for public health.

Periodontitis is directly preceded by a dynamic, polymicrobial oral microbiome. As a bacterial community develops, 
the ecological succession from a microbial community to a state of dysbiosis manifests as emergence of newly dominant community members rather than the appearance of novel species [5]. The specific groups and combinations of bacteria, including the "red complex," Porphyromonas gingivalis (P. gingivalis), Treponema denticola (T. denticola), and Tannerella forsythensis ( $T$. forsythensis), have been strongly associated with the pathology of periodontitis [6]. These bacteria can alter host immune competence with increased production of virulence factors. For example, the $P$. gingivalis virulence factors have been shown to decrease the host response [7] by subverting innate immune signaling. Though the relationship with the host is normally homeostatic, by manipulating the crosstalk between complement and Toll-like receptors (TLRs) [8], a destructive change is triggered [9]. In addition, the virulence factors of $P$. gingivalis can specifically recognize epitope-specific CD4+ $\mathrm{T}$ cell phenotypes [10]. Furthermore, a cluster of species with a less stringent association with disease was defined as the "orange complex" and includes Prevotella spp., Fusobacterium spp., and Parvimonas micra (formerly Peptostreptococcus micros) [11]. They were also found to be associated with immunity. Fusobacterium nucleatum (F. nucleatum) could have the capacity to induce a downregulation of antimicrobial peptides, such as $\mathrm{h} \beta \mathrm{D}-1$ and LL-37, but this downregulation of the host defense may be another bacteria-mediated virulence mechanism [12].

Previous studies have suggested that both host genetic and immunological factors are important in oral microbiome dysbiosis, further demonstrating the complex nature of this condition. Race/ethnicity, psychosocial stress, socioeconomic status, gender, and other sociodemographic factors are also gaining more and more importance in the incidence and severity of periodontitis and alterations to oral microbiomes [13]. However, the influence of gender in the process remains controversial. No sex relationship was found for yeasts and staphylococci in a microbiological analysis of 3075 "refractory" periodontitis patients [14]. Similarly, a study that analyzed the relationship of gender and race with components of the subgingival microflora from individuals with different degrees of periodontal disease and periodontal health found no significant differences between males and females [15]. In contrast, Umeda et al. suggested an association between gender and carriage of a specific organism, demonstrating that Prevotella intermedia/nigrescens ( $P$. intermedia/P. nigrescens) is more likely to be found in the saliva and subgingival and supragingival plaques of males than females [16].

In the current study, we identified sex-specific differences in the oral microbiomes of youths with severe periodontitis through $16 \mathrm{~S}$ rRNA gene sequencing and predicted the variation of the oral microbiomes that may be related to immunity in order to gain further understanding of periodontitis.

\section{Materials and Methods}

2.1. Participants and Inclusion Criteria. We enrolled a total of 17 participants (11 males and 6 females) who visited the
TABle 1: Age information of the enrolled participants.

\begin{tabular}{lcc}
\hline Group & Number & Age \\
\hline Group 1 & 11 & $35.73 \pm 5.93$ \\
Group 2 & 6 & $34.17 \pm 7.49$ \\
$P$ & & 0.643 \\
\hline
\end{tabular}

medical examination center at The Second Xiangya Hospital of Central South University. The inclusion criteria were patient age between 20 and 44 years; the presence of at least 15 existing natural teeth (excluding third molars); no removable partial denture, bridge, or implant; no antibiotic use within 1 month and no periodontal treatment within the last 6 months; no other bacterial infectious oral disease or systemic disease; and the patient was a nonsmoker. All of the participants received a comprehensive oral examination, which included a professional assessment by a specialized dentist based on the standards of the 2017 World Workshop on the Classification of Periodontal and Peri-Implant Diseases and Conditions "Staging and Grading of Periodontitis: Framework and Proposal of a New Classification and Case Definition" [17]. Before measurement, the sites were airdried. "Initial" periodontitis was defined as at least one tooth with probing depth $(\mathrm{PD}) \geq 3 \mathrm{~mm}$ and attachment loss (AL) $\geq 3 \mathrm{~mm}$ or $\mathrm{PD} \geq 4 \mathrm{~mm}$ and $\mathrm{AL} \geq 3 \mathrm{~mm}$ in $\leq 30 \%$ of teeth. "Moderate" periodontitis was defined as $\mathrm{PD} \geq 5 \mathrm{~mm}$ and $\mathrm{AL} \geq 4 \mathrm{~mm}$ in $<30 \%$ of teeth or $\mathrm{PD} \geq 4 \mathrm{~mm}$ and $\mathrm{AL} \geq 3 \mathrm{~mm}$ in $30-60 \%$ of teeth. "Severe" periodontitis was PD $\geq 5 \mathrm{~mm}$ and $\mathrm{AL} \geq 4 \mathrm{~mm}$ in $\geq 30 \%$ of teeth or $\mathrm{PD} \geq 4 \mathrm{~mm}$ and $\mathrm{AL} \geq$ $3 \mathrm{~mm}$ in $\geq 60 \%$ of teeth. Patients with severe (stage III) and advanced (stage IV) periodontitis were recruited [18]. The participants were divided into two groups based on gender. The study was approved by the Ethics Committee of The Second Xiangya Hospital of Central South University. All participants were informed of the research aims and provided verbal and written consent. The clinical trial registration number is ChiCTR2100046828.

2.2. Subgingival Plaque Collection. Patients were sampled after the oral examination. Supragingival plaque was removed carefully before prior to sampling. The subgingival plaque was collected using a sterile Gracey curette. The curette was introduced into the bottom of the site, and the plaque content was removed in a single stroke into a $1.5 \mathrm{~mL}$ tube containing $1 \mathrm{~mL}$ of PBS. Samples were stored at $-80^{\circ} \mathrm{C}$.

\subsection{DNA Extraction and $16 S$ rRNA Gene Library} Preparation and Sequencing. Genomic DNA was isolated from each sample using the DNeasy PowerSoil Kit (QIAGEN) following the manufacturer's instructions. We assessed the yield, purity, and integrity of the DNA using a NanoDrop 2000 spectrometer (Thermo Fisher Scientific Inc., MA USA) and agarose gel electrophoresis as appropriate. 16S rRNA was completed by oebiotech (Shanghai, China). 16S rRNA gene amplification was performed in two steps. First, the V3-V4 hypervariable region was amplified by PCR using the genomic DNA and the following 


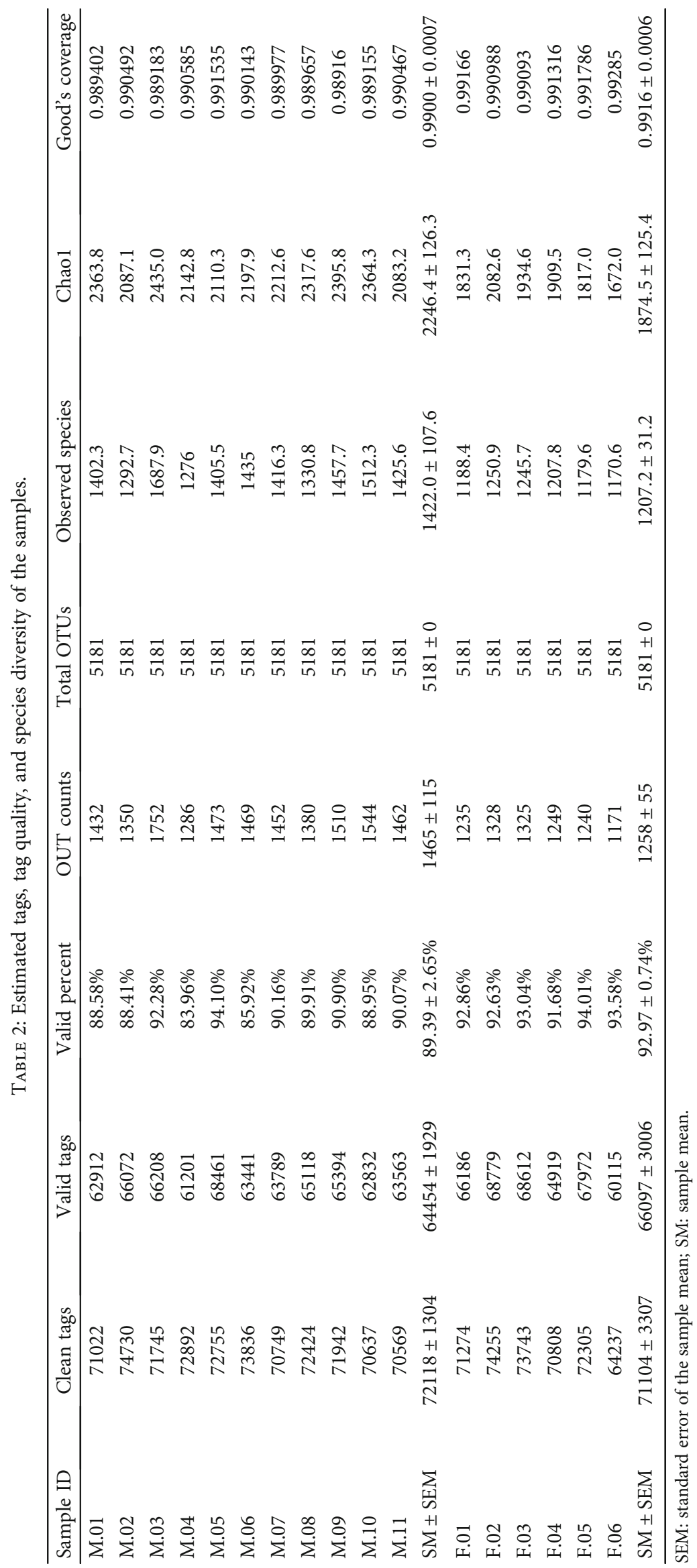




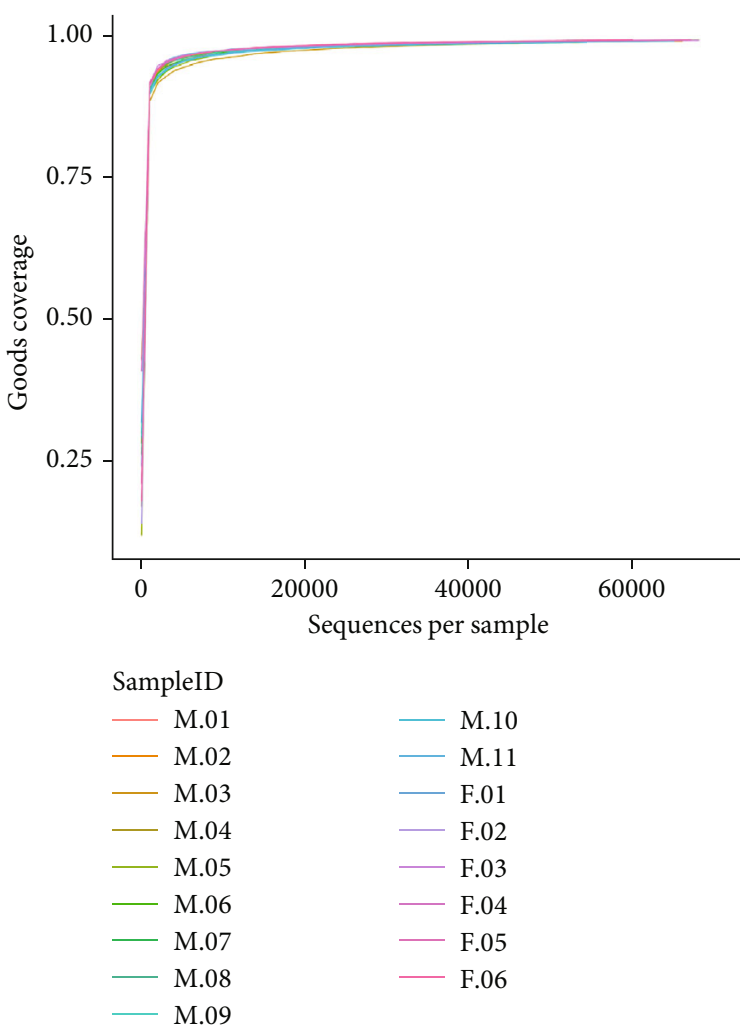

(a)

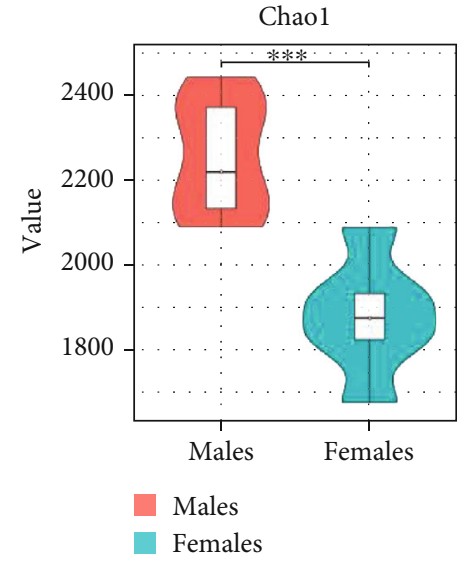

(c)

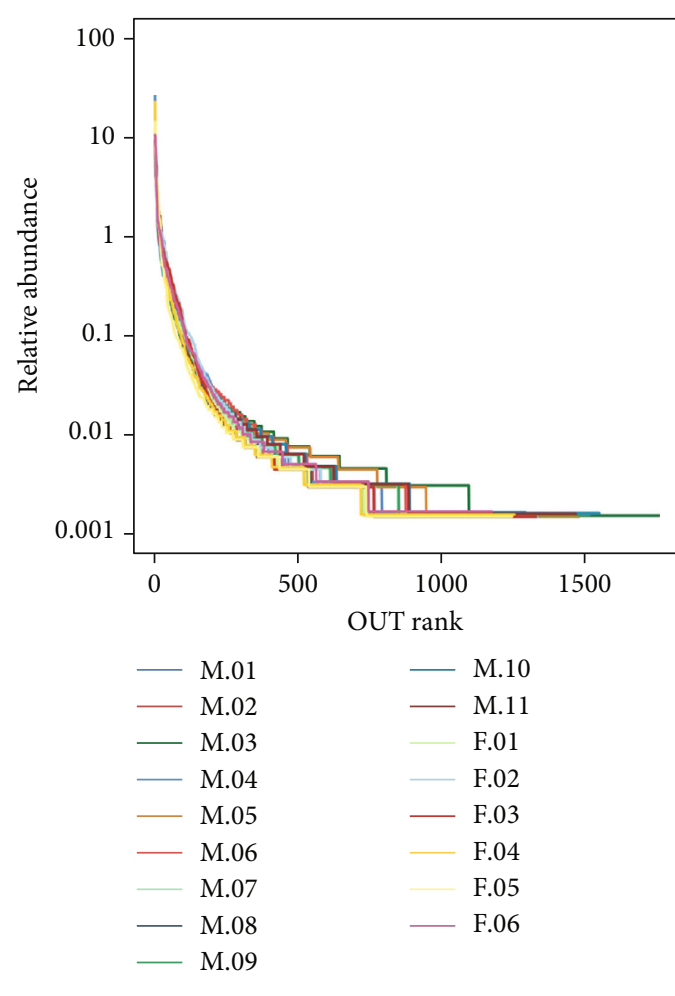

(b)

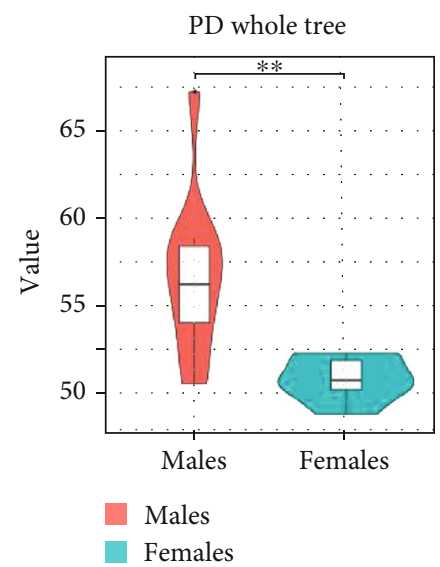

(d)

Figure 1: Continued. 


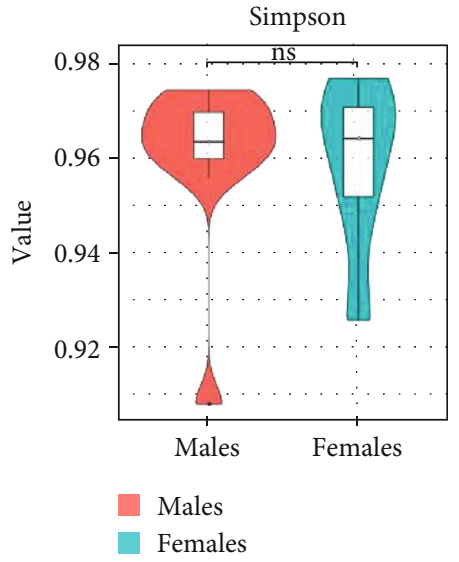

(e)

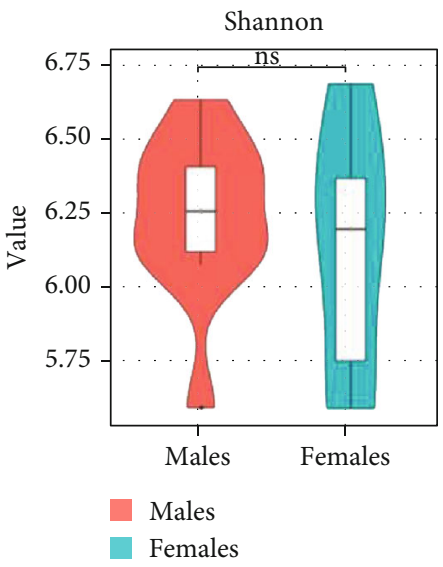

(f)

FIGURE 1: Sequence quality and $\alpha$-diversity analysis of samples from young male and female patients with severe and advanced periodontitis. (a) Rarefaction curves based on Good's coverage for all samples from males and females. The horizontal axis shows the number of operational taxonomic units (OTUs), and appropriate number of sequences is shown on the vertical axis. (b) Rank-abundance curve representative of all samples from males and females. (c-f) Violin plots comparing $\alpha$-diversity indices Chao1, PD whole tree, Simpson index, and Shannon index between the two groups. ${ }^{* * *} P<0.001 ;{ }^{* *} P<0.01$; ns: not significant.

primers: forward, $343 \mathrm{~F}\left(5^{\prime}\right.$-TACGGRAGGCAGCAG-3'); reverse, $798 \mathrm{R}\left(5^{\prime}\right.$-AGGGTATCTAATCCT-3'). Amplification was performed in a reaction mixture containing $15 \mu \mathrm{L}$ of $2 \mathrm{x}$ Gflex PCR Buffer, $5 \mu \mathrm{M}$ primer $343 \mathrm{~F}, 5 \mu \mathrm{M}$ primer $798 \mathrm{R}, 0.75 \mathrm{U}$ Tks Gflex DNA Polymerase (Takara), and $50 \mathrm{ng}$ of template DNA in a total volume of $30 \mu \mathrm{L} /$ sample. Reactions were run in a PCR thermocycler (BIO-RAD) according to the following cycling program: $5 \mathrm{~min}$ of denaturation at $94^{\circ} \mathrm{C}$, followed by 26 cycles of $30 \mathrm{~s}$ at $94^{\circ} \mathrm{C}$ (denaturing), $30 \mathrm{~s}$ at $55^{\circ} \mathrm{C}$ (annealing), and $20 \mathrm{~s}$ at $72^{\circ} \mathrm{C}$ (elongation), with a final extension at $72^{\circ} \mathrm{C}$ for $5 \mathrm{~min}$. The amplified products were checked by $1 \%$ agarose gel electrophoresis, purified using AMPure XP beads (Agencourt), and amplified in another round of PCR.

In the second step, sequencing primers and adaptors $(1 \mathrm{x}$ KAPA HiFi Hotstart ReadyMix, $0.5 \mu \mathrm{M}$ fusion forward and $0.5 \mu \mathrm{M}$ fusion reverse primer, and $30 \mathrm{ng}$ Meta-gDNA) were added to $2 \mu \mathrm{L}$ of the diluted amplicons to a total volume of $50 \mu \mathrm{L}$. The PCR was run as described above except with 7 cycles. The Qubit quantification system (Life Technologies) was used for quantification of amplicons according to the manufacturer's instructions. In a single tube, the amplification products were pooled in equimolar amounts and the concentration was determined using the Qubit system. Amplicons were sequenced on the Illumina MiSeq System (Illumina Inc., CA, USA).

2.4. Bioinformatic and Statistical Analysis. Fastq files were demultiplexed using MiSeq Controller Software (Illumina Inc.). Vsearch (v. 2.4.2) [19] was used for operational taxonomic unit (OUT) clustering at or above 97\%. The taxonomy of the OTUs was assigned, and sequences were aligned according to the RDP classifier Naive Bayesian method [20] and the Silva database. The OTUs were analyzed by phylogenetic methods in the Quantitative Insights into Microbial Ecology (QIIME) software (v.1.9.0). We calculated the $\alpha$-diversity (observed species number, Shannon index, Simpson index, Chao index, PD whole tree index, and Good's coverage index) and $\beta$-diversity (binary Jaccard, unweighted UniFrac distances, and weighted UniFrac distances) based on the rarefied OTU counts. We used the principal component analysis (PCA) and principal coordinate analysis (PCoA) to plot the similarity or difference in the composition of the sample community. Sequences were used for microbial community metagenome prediction with PICRUSt [21] based on the GreenGenes database [22]. The differential taxa analyses were performed with the linear discriminant analysis effect size (LEfSe) using default parameters (the significance threshold of alpha parameter is set to 0.05 , and the logarithmic LDA score cutoff value is set to 2.0). Functional inference was identified using Kyoto Encyclopedia of Gene and Genomes (KEGG) pathways. The Wilcoxon test was used to determine differentially abundant KEGG pathways between the two groups.

\section{Results}

3.1. Clinical Characteristics of Participants. The mean \pm standard deviation (SD) patient age was $35.73 \pm 5.93$ and $34.17 \pm 7.49$ for males and females, respectively. There was no statistically significant difference in age between males and females $(P>0.05$, Table 1$)$.

3.2. Sequence Information. We used Illumina MiSeq and QIIME to analyze the microbiome composition in subgingival plaques from patients with severe and advanced periodontitis. An average of 72,118 (min. 70,569, max. 74,730) clean tags was generated from males and 71,104 (min. 64,237, max. 74,255) clean tags from females. After removing chimeras, the number of analyzed tags decreased to an average of 64,454 (min. 61,201, max. 68,461) in males and 66,097 (min. 60,115, max. 68,779) in females. Using Vsearch (v.2.4.2) software to cluster individual sequences with $97 \%$ genetic similarity at the species level, we identified 5181 


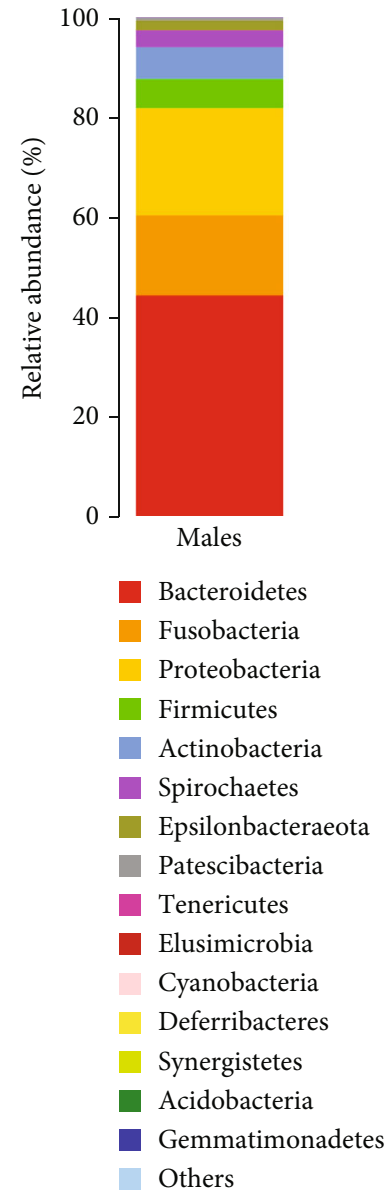

(a)

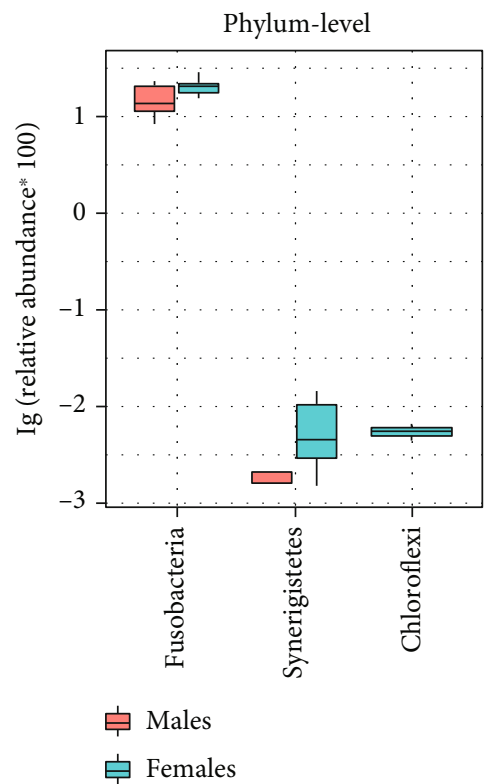

官 Females

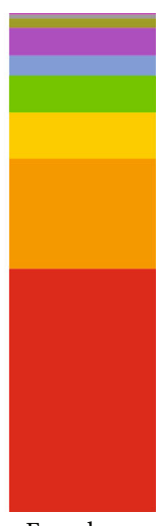

Females

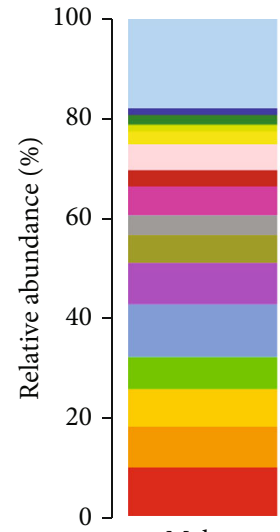

Males

Prevotella

Fusobacterium

Leptotrichia

Porphyromonas

Neisseria

Capnocytophaga

Prevotella_7

Prevotella_2

Corynebacterium

- Treponema_2

Aggregatibacter

Alloprevotella

- Tannerella

- Campylobacter

F0058

Others

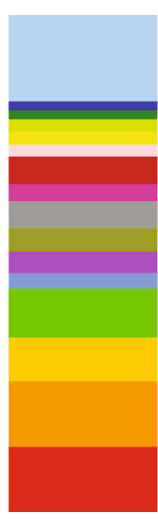

Females

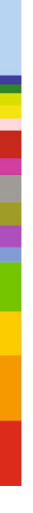

(b)

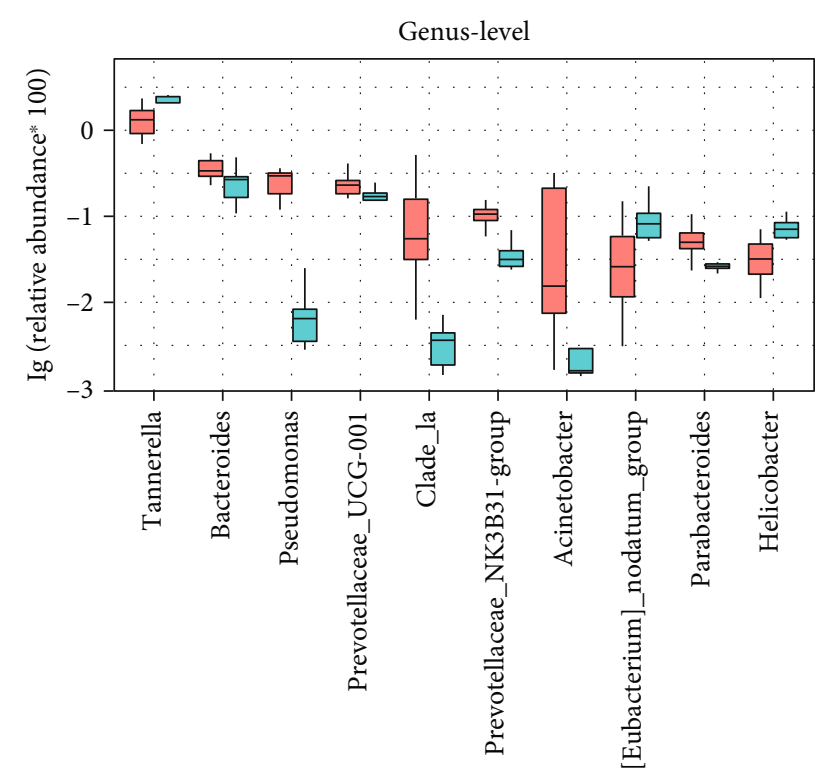

官 Males

官 Females

(c)

(d)

FIgUre 2: The oral microbiome composition of young male and female patients with severe and advanced periodontitis. (a) Phylum level composition. (b) Genus level composition. Species difference analysis between males and females by the Kruskal-Wallis test at the level of the (c) phylum and (d) genus. 
OTUs among 17 samples, with a minimum of 1171 OTUs in a single sample and a maximum of 1752 OTUs (Table 2).

The quality of sequencing can generally be determined based on the index of the rarefaction curve, rankabundance curve, or species accumulation curve. In our sample, the rarefaction curves based on Good's coverage tended to be close to saturated, indicating that the number of samples was ample, and there were no obvious differences in the rarefaction curves between the two groups (Figure 1(a)). Similar results were obtained with the rankabundance curves, which indicate that the amount of sequencing data was abundant enough to reflect most of the microbial species information in the sample (Figure 1(b)).

3.3. Diversity Analysis. $\alpha$-Diversity reflects the richness and diversity of oral microbial communities. Bacterial richness and evenness within each group ( $\alpha$-diversity) was estimated using the Chaol index, PD whole tree measurements, and the Simpson and Shannon indices (Figures 1(c)-1(f)). The Chaol index and PD whole tree were significantly higher in males than females (Wilcoxon rank-sum test, $P<0.01$ ). The Simpson and Shannon indices were not significantly different between the two groups (Wilcoxon rank-sum test, $P>0.05)$. Thus, the community diversity was not different in patients with severe and advanced periodontitis according to gender.

Subsequently, for community structure, relative abundance was assessed at the top 15 phylum (Figure 2(a)) and genus (Figure 2(b)) level, respectively. Although the dominant florae were similar, the abundance of each species was vastly different. The main microbiota $(>1 \%)$ at the phylum and genus level and their abundance are listed in Table 3. Statistical analyses of the major bacteria between the two groups were carried out, and boxplots representing the abundance of the top 3 different species at the phylum and top 10 different species at the genus levels $(P<0.05)$ are shown in Figures 2(c) and 2(d). Compared to males, the relative abundance of Fusobacteria, Synergistetes, and Chloroflexi at the phylum level increased in females $(P<0.05)$, in which the abundance of Chloroflexi in males is 0 (Figure 2(c)). At the genus level, the relative abundance of Tannerella, Eubacterium_nodatum_group, and Helicobacter was higher in females than in males $(P<0.05)$ while the relative abundance of Bacteroides, Pseudomonas, Prevotellaceae_UCG-001, Clade_la, Prevotellaceae_NK3B31-group, Acinetobacter, and Parabacteroides in females was lower than that in males $(P<0.05$; Figure $2(\mathrm{~d}))$. Figure 3 shows the Wilcoxon analysis of differences between males and females in the relative proportions at the phylum (Figure 3(a)) and genus (Figure 3(b)) levels in the form of a heat map.

$\beta$-Diversity reflects the differences of microbial diversity between two groups. Therefore, we evaluated the extent of the similarity between the microbial communities using PCA, PCoA, and nonmetric multidimensional scaling (NMDS) analysis based on unweighted UniFrac distances. Males and females with severe and advanced periodontitis could form relative clusters (Figures $4(\mathrm{a})-4(\mathrm{c})$ ) and generally
TABLE 3: The main microbiota $(>1 \%)$ at the phylum and genus level and their abundance.

\begin{tabular}{lccc}
\hline Level & Microbiota & Males & Females \\
\hline \multirow{4}{*}{ Phylum level } & Bacteroidetes & $44.23 \%$ & $48.77 \%$ \\
& Fusobacteria & $15.92 \%$ & $22.01 \%$ \\
& Proteobacteria & $21.57 \%$ & $9.29 \%$ \\
& Firmicutes & $5.83 \%$ & $7.36 \%$ \\
& Actinobacteria & $6.46 \%$ & $4.07 \%$ \\
& Spirochaetes & $3.26 \%$ & $5.56 \%$ \\
& Epsilonbacteraeota & $1.97 \%$ & $1.87 \%$ \\
& Prevotella & $9.89 \%$ & $13.29 \%$ \\
& Fusobacterium & $8.25 \%$ & $13.16 \%$ \\
& Leptotrichia & $7.6 \%$ & $8.78 \%$ \\
& Porphyromonas & $6.35 \%$ & $9.87 \%$ \\
Neisseria & $10.63 \%$ & $4.07 \%$ \\
& Capnocytophaga & $8.31 \%$ & $4.43 \%$ \\
& Prevotella_7 & $5.62 \%$ & $4.72 \%$ \\
Prevotella_2 & $3.99 \%$ & $5.42 \%$ \\
& Corynebacteriumand & $5.72 \%$ & $3.35 \%$ \\
Treponema_2 & $3.26 \%$ & $5.56 \%$ \\
& Aggregatibacter & $5.23 \%$ & $2.34 \%$ \\
Alloprevotella & $2.6 \%$ & $2.69 \%$ \\
Tannerella & $1.37 \%$ & $2.43 \%$ \\
& Campylobacter & $1.92 \%$ & $1.79 \%$ \\
& F0058 & $1.32 \%$ & $1.79 \%$ \\
\hline & & & \\
& & &
\end{tabular}

be separated into a two-dimensional spatial distribution, indicating that the two communities were different (Figures 4(b) and 4(c)). An ANOSIM test of the $\beta$-diversity demonstrated a significant difference $(R$ value with the unweighted UniFrac distance matrix $=0.4515 ; P=0.001)$.

3.4. LEfSe Analysis. The linear discriminant analysis effect size (LEfSe) was used to identify taxa characterizing the differences between the two groups. Based on the results of the species-abundance comparison between the two groups of oral microbiotas, the community differences between groups were analyzed at the phylum to genus level using LEfSe analysis (Figures 5(a) and 5(b)). Our results suggest that the phylum Fusobacteria, its class Fusobacteria, and its order Fusobacteriales, along with the family Tannerellaceae and its genus Tannerella, were abundant in the female patients. In addition, the order Pseudomonadales and its family Pseudomonadaceae and its genus Pseudomonas, along with the genus Papillibacter, were abundant in the male patients. Tannerella was the key component that could make the difference between groups by selecting the first 30 genera of relative abundance and carrying out the random forest feature selection procedure (Figure 5(c)).

3.5. Functional Metabolism Pathway Prediction. Among the KEGG pathways predicted for microbial function, we identified immune-related pathways that exhibit a significant difference in abundance between males and females at 


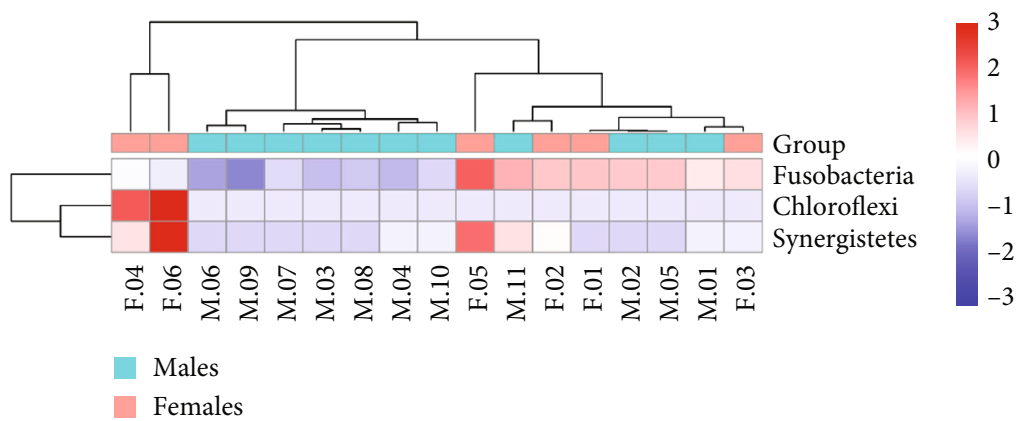

(a)

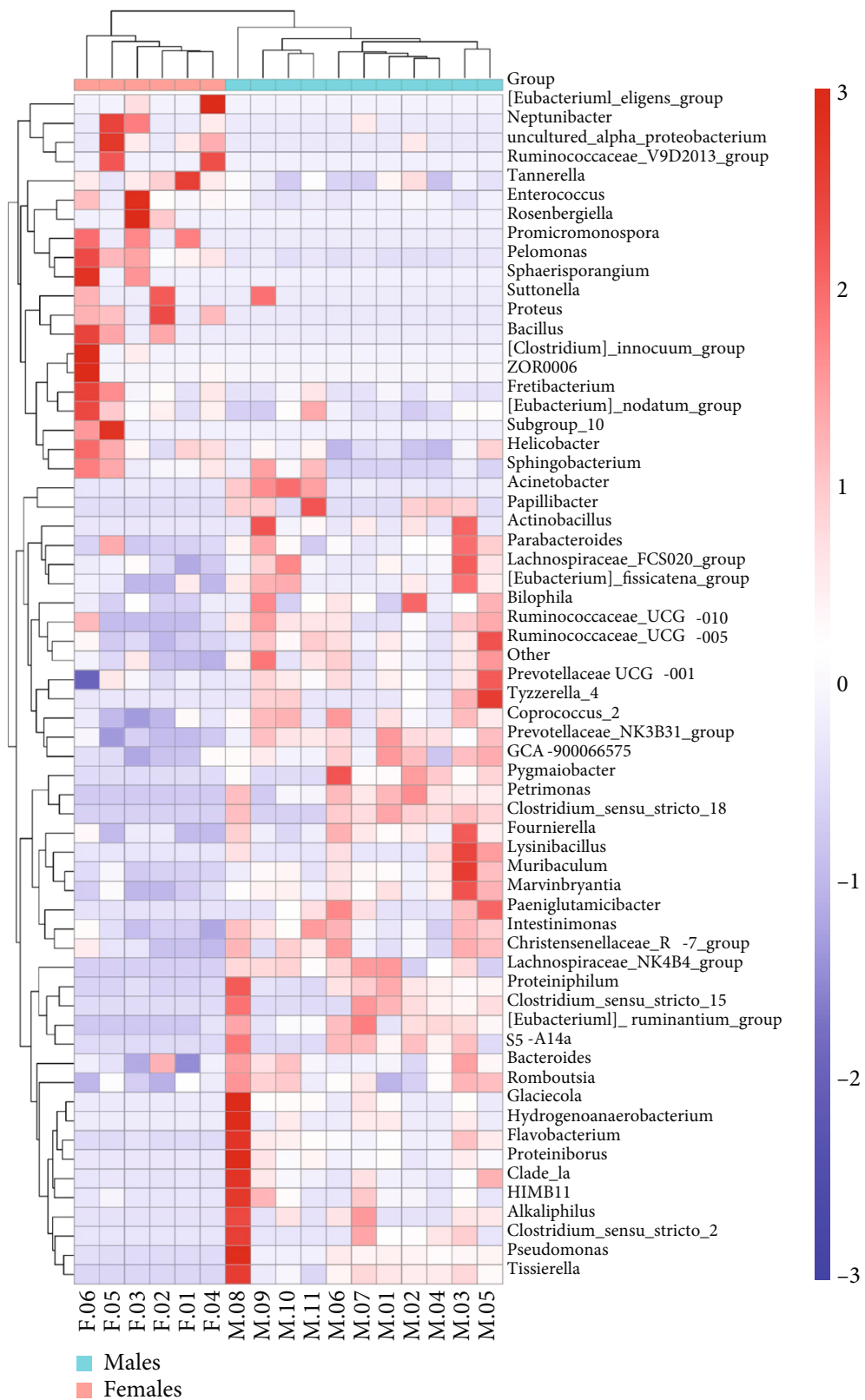

(b)

Figure 3: Heat map of the differential oral microbiomes between young male and female patients with severe and advanced periodontitis. (a) Phylum level. (b) Genus level. The horizontal axis is the sample information (group and number), and the vertical axis is the species annotation. Colors indicate the Spearman rank correlation. 


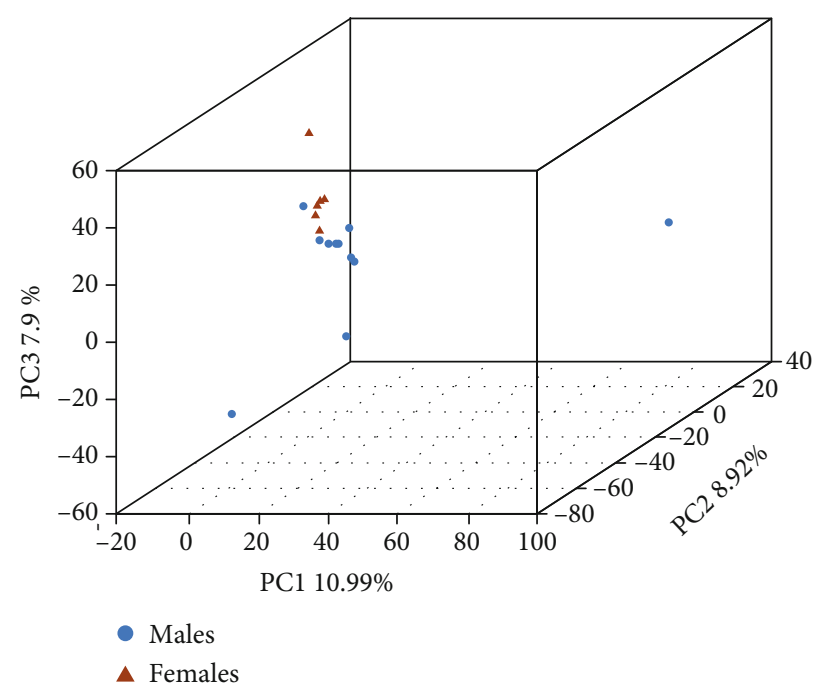

(a)

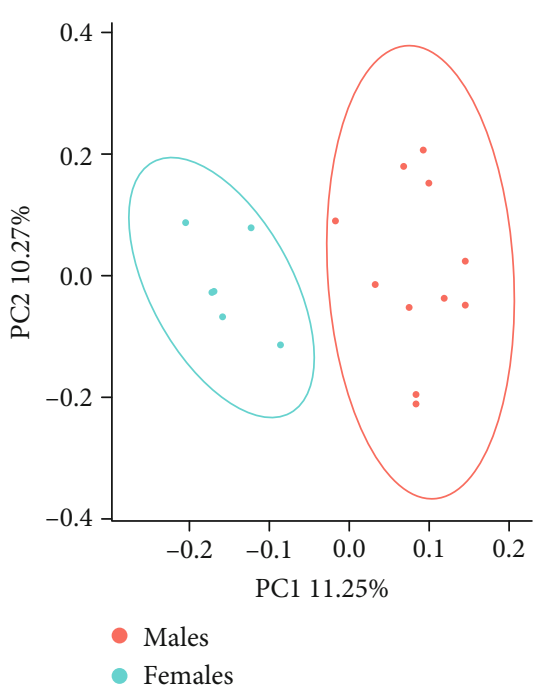

(b)

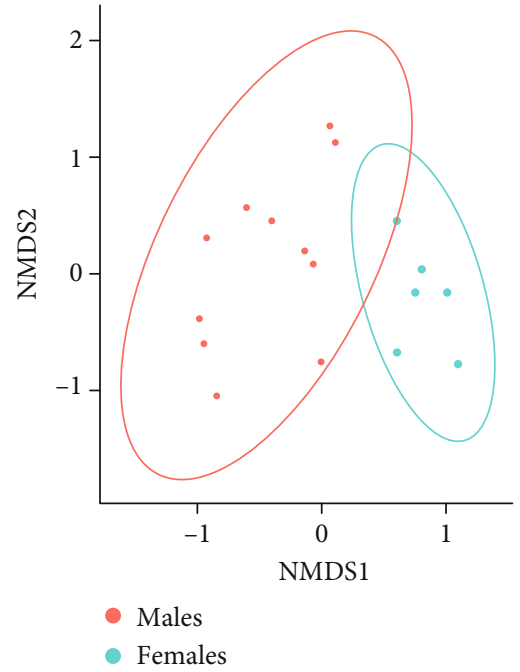

(c)

FIGURE 4: $\beta$-Diversity analysis of young male and female patients with severe and advanced periodontitis. (a) The similarity of the microbial communities between males and females was analyzed by principal component analysis, (b) principal coordinate analysis, and (c) nonmetric multidimensional scale (NMDS) based on the unweighted UniFrac distance.

different levels in Wilcoxon tests (Figure 6). The pathway analysis of the predicted KEGG pathways in each sample at the phylum level indicated that the microbiomes with altered abundance are mainly involved in pathways related to the immune system and cardiovascular disease (Figure 6(a)). The results at the class level also showed that the pathways were related to the immune system, including the NOD-like receptor signaling pathway and antigen processing and presentation (Figure 6(b)). The predicted KEGG pathways based on group showed that the immune system was also significantly enriched in females and immune system diseases concentrated in males (Figure 6(c)).

\section{Discussion}

In this study, we used 16S rRNA high-throughput sequencing to determine the differences in species abundance in the oral microbiomes of young men and women with severe periodontitis. Regarding $\alpha$-diversity based on species richness, males had higher values than females. In addition, $\beta$ diversity suggested that the samples were divided into reasonable groups. The Kruskal-Wallis test based on the relative abundance of species composition, combined with LEfSe analysis showed that the dominant bacteria in males were Pseudomonas and Papillibacter, whereas the dominant bacteria in women were the order Fusobacteriales and the genus Tannerella. KEGG analysis predicted that the variation in the oral microbiome may be related to the immune system in women, whereas immune system diseases are the dominant pathway in men, offering further understanding of periodontitis.

Periodontitis is an inflammation that extends deep into tissue and leads to the loss of supporting connective tissue and alveolar bone [23]. Oral microbiomes are defined as 


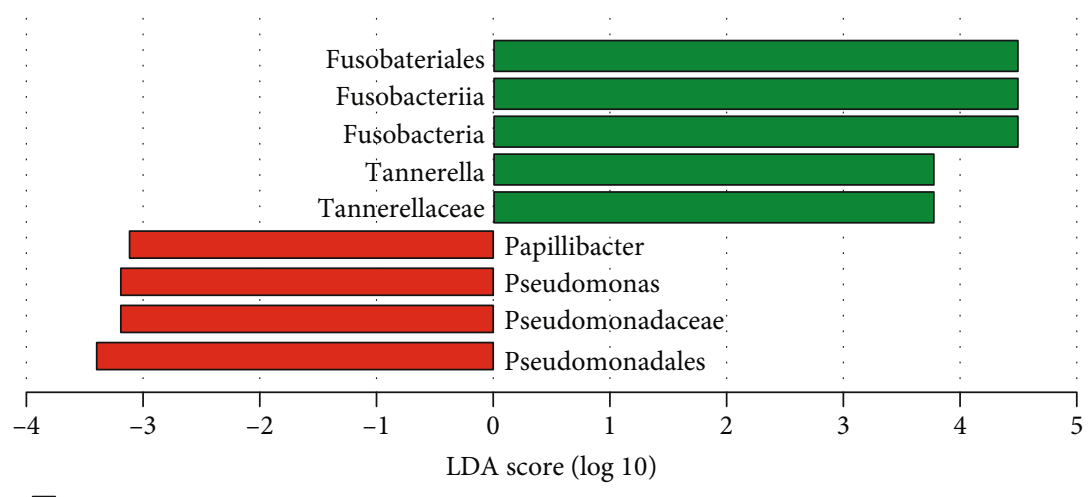

Males

Females

(a)

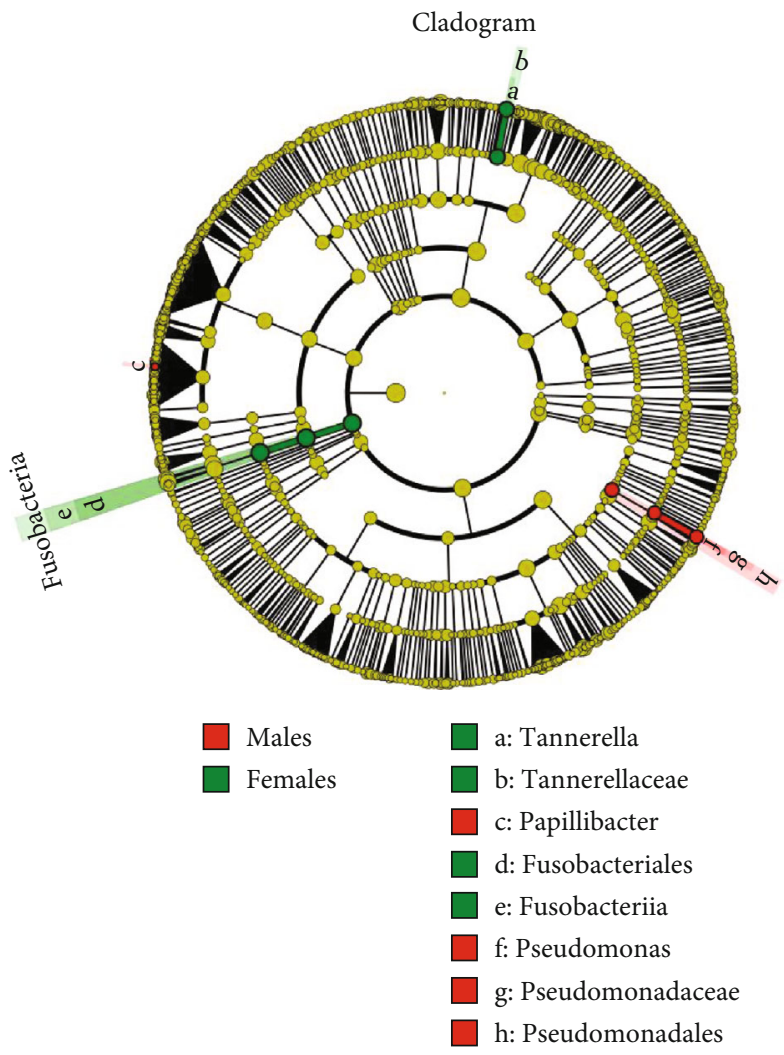

(b)

Figure 5: Continued. 


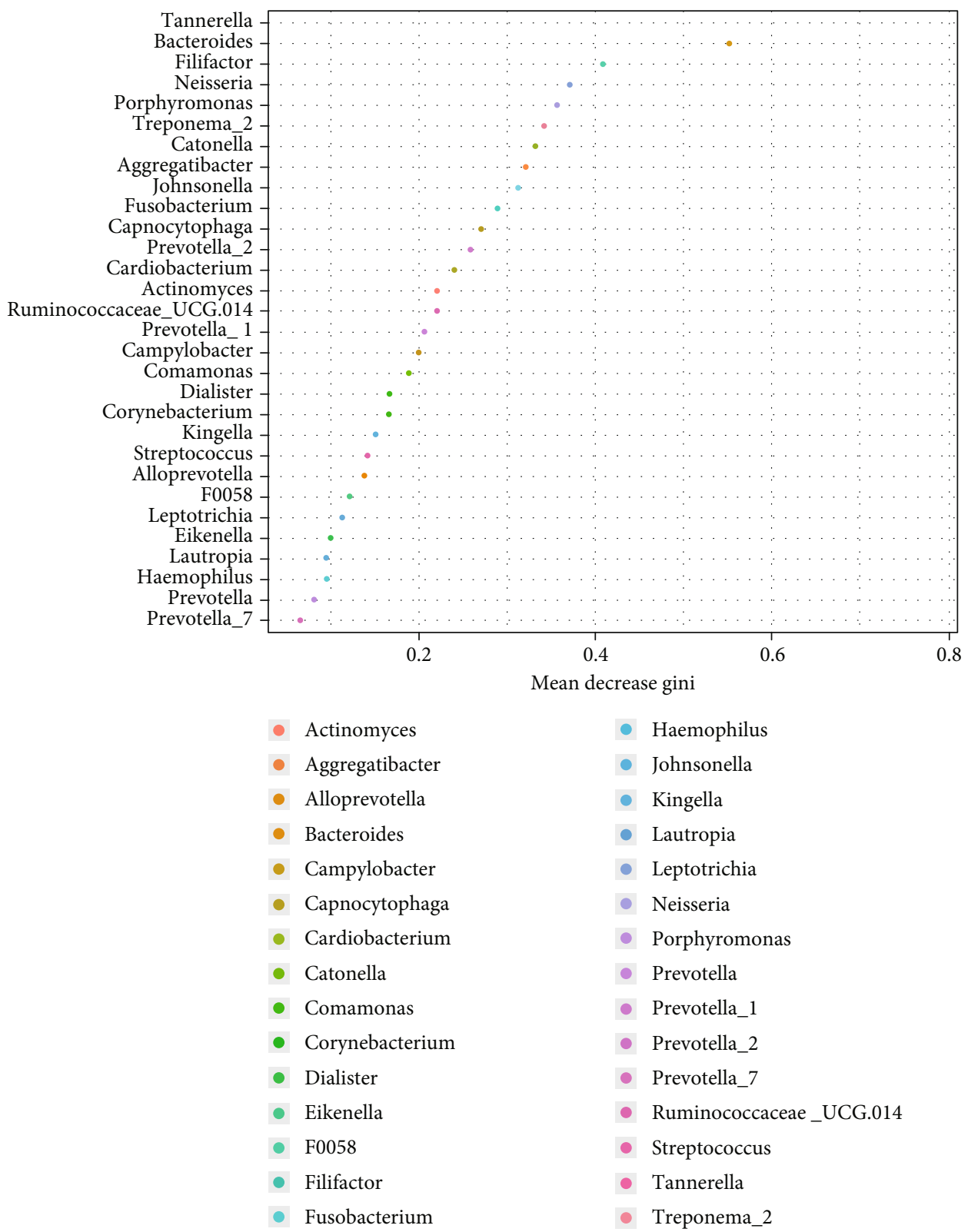

(c)

FIGURE 5: Linear discriminant analysis (LDA) effect size (LEfSe) analysis of the oral microbiomes in young male and female patients with severe and advanced periodontitis. (a) Histogram of the LDA scores of males and females. Female-enriched taxa are indicated by a positive LDA score (green), and taxa enriched in males have a negative score (red). (b) Taxonomic cladogram obtained from LEfSe analysis. Red indicates male, green indicates female, and yellow indicates nonsignificant between males and females. (c) Point map of species importance. The horizontal axis is the measure of importance, and the vertical axis is the name of the species sorted by importance.

the microorganisms found in the oral cavity, including bacteria, viruses, fungi, protozoa, and archaea [24]. The dysbiosis hypothesis states that the transition from periodontal health to disease occurs due to changes in species abundance among the bacteria in the periodontal pocket. This shift in the composition of the microbial community is sufficient to alter the host-microbe crosstalk, resulting in destructive inflammation and bone loss [7]. The healthy oral microbial community usually has low diversity and richness [25]. The species diversity of the general flora is lower in youth than in the elderly, which is often reflected in the periodontal condition of young people being healthier. In addition, young people are more affected by hormones, so there is clinical significance to discussing gender differences. We found that the Chaol index and PD whole tree were significantly higher in males than females, representing the greater bacterial richness in men, and that they may have severe periodontal tissue destruction. An investigation of the gut microbiota in healthy Japanese subjects found that, although there were significant differences in the microbial structure 


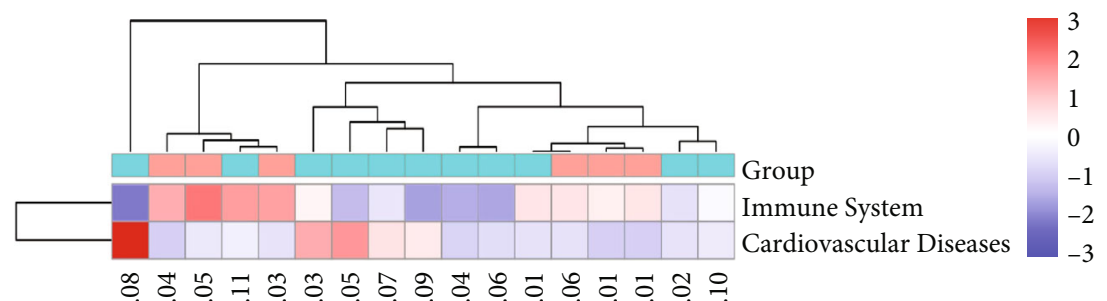

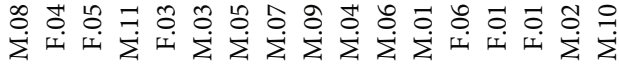

$\square$ Males

$\square$ Females

(a)

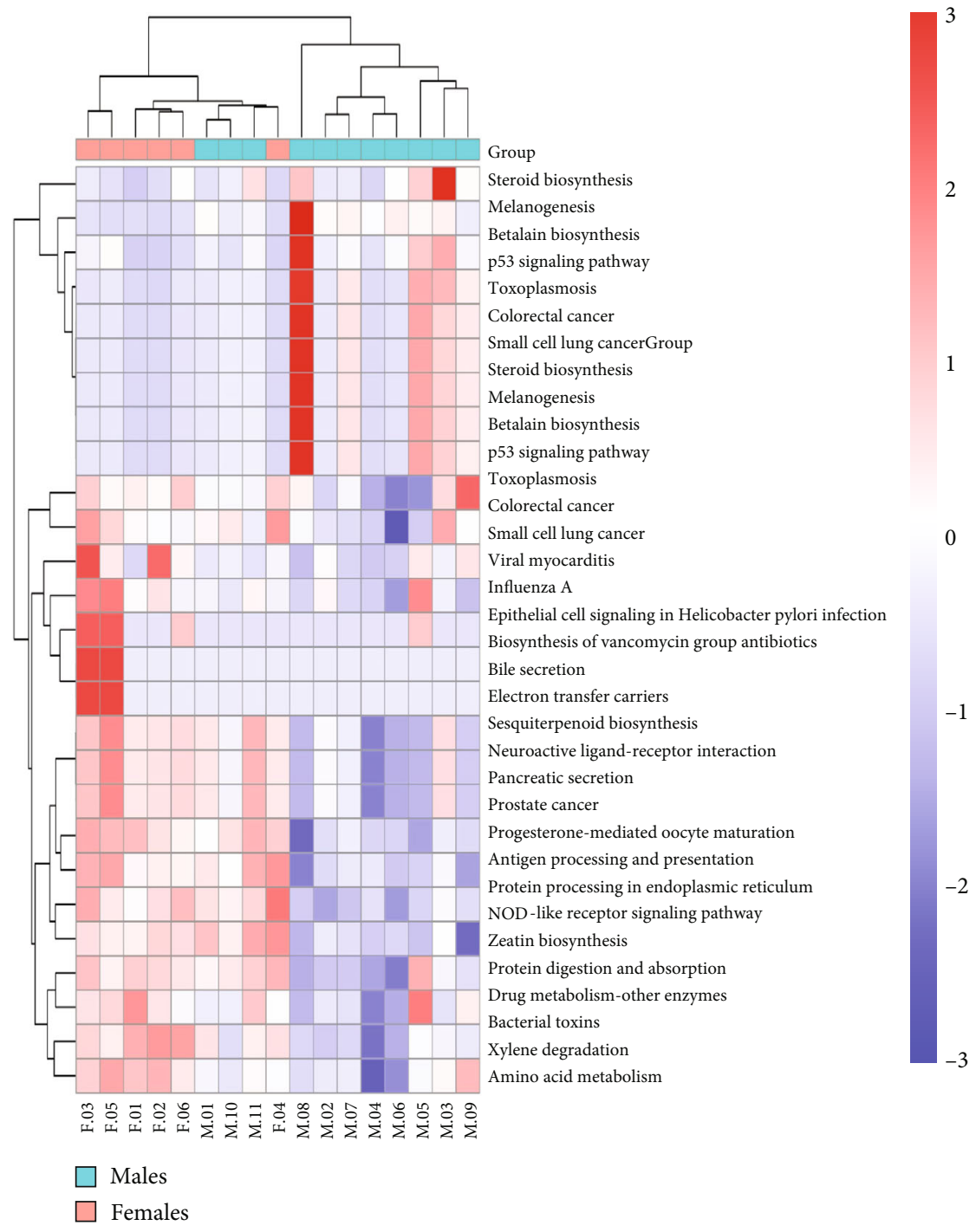

(b)

FIgUre 6: Continued. 


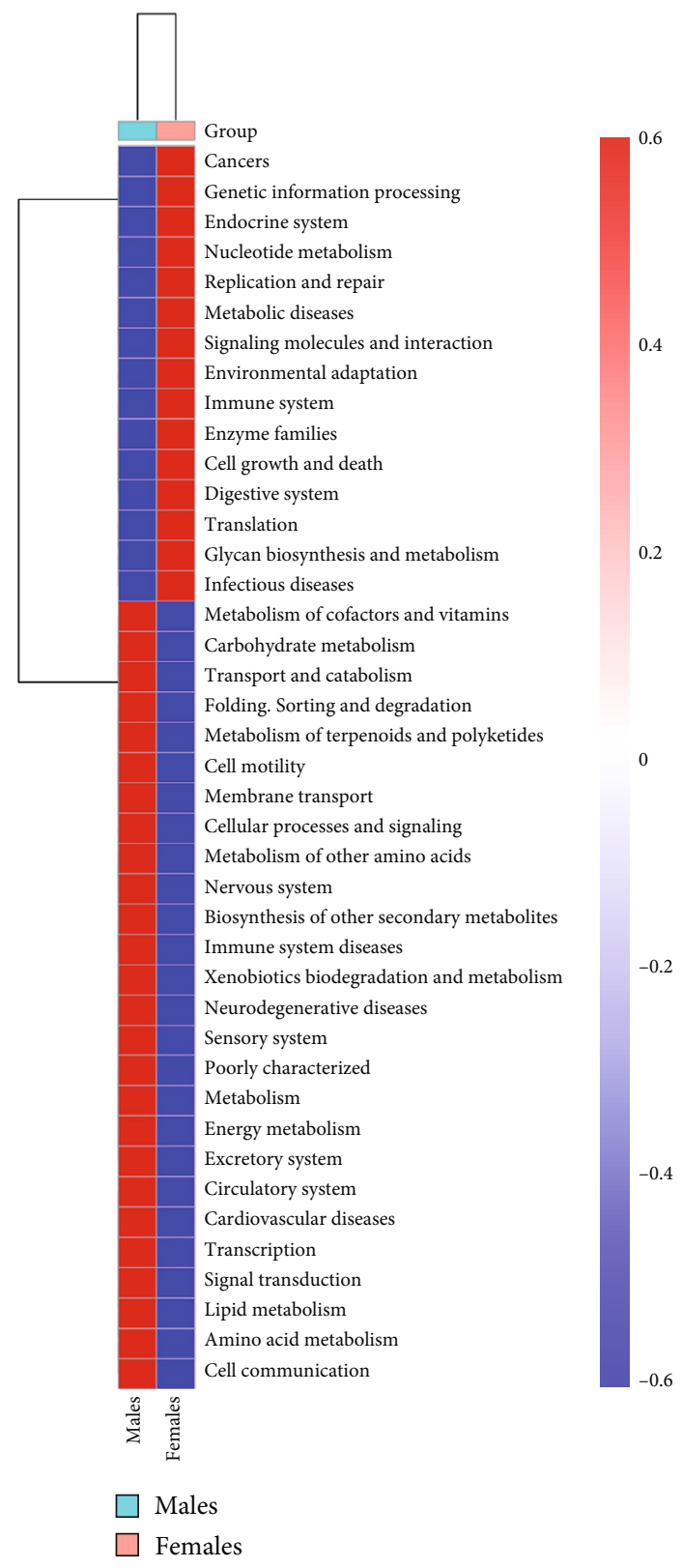

(c)

FIGURE 6: Pathway enrichment analysis based on KEGG. (a) Heat maps of differential pathways at the phylum level and the class level of KEGG in individual patients. (c) Heat map of differential pathways between males and females at the phylum level of KEGG.

between males and females aged $20-89$ years, the $\alpha$-diversity of the gut microbiota was not different between males and females or among age groups [26].

At the American Academy of Periodontology Workshop held in 1996, experts agreed there are 11 microorganisms that are closely related to periodontal disease, including gram-negative bacteria such as $P$. gingivalis, $T$. forsythia, $P$. intermedia/P. nigrescens, F. nucleatum, Aggregatibacter actinomycetemcomitans (A.actinomycetemcomitans), and $T$. denticola [27]. Among them, we found that Fusobacteriales are significantly higher in women than in men through the Kruskal-Wallis test at the level of phylum and LEfSe analysis. The most prevalent in females was Fusobacteria, anaero- bic gram-negative bacilli that cause tissue necrosis, septicemia, intra-amniotic infections, premature labor, and disorders of the oral cavity, such as pulpal infections, alveolar bone abscesses, and periodontal disease. F. nucleatum induces production of inflammatory cytokines and cell proliferation and inhibits apoptosis, cellular invasion, and migration through host cell genomic alterations [12]. F. nucleatum reacts to the inflammatory response during periodontal disease and induces secretion of salivary antimicrobial peptides that have an impact on host cells and modulate the immune response [12]. Kostic et al. have indicated that Fusobacteria generates a proinflammatory microenvironment conducive to the progression of colorectal neoplasia 
by recruiting tumor-infiltrating immune cells [28]. The phylum Proteobacteria was enriched in male patients. Guan et al. have found that high expression is also present in severe bronchiectasis [29].

Our results showed significant variation in the oral microbiomes of the youths of different genders. In our research, at the genus level, the increased Porphyromonas, Prevotella, and Tannerella in the oral microbiomes of females contributed to the periodontal destruction. P. gingivalis is the most extensively studied of all major periodontal pathogenic organisms. The virulence factor of $P$. gingival is lipopolysaccharides (LPS), fimbriae, and capsule [30]. P. gingivalis LPS and fimbriae stimulation led to the upregulation of TLR2 expression and proinflammatory cytokine production in vivo and in vitro, resulting in pathogenic inflammatory bone loss [31]. P. intermedia and P. nigrescens are often isolated from periodontal sites and were once considered to be two different genotypes of $P$. intermedia [32, 33]. P. intermedia LPS can participate in bone destruction by stimulating the differentiation and activity of osteoclasts and promoting the release of matrix metalloproteinases from osteoclasts and osteoblasts [34]. It is worth noting that we found that Tannerella is significantly increased in women through the LEfSe analysis. Tannerella is gram-negative, obligate anaerobic, nonmotile, pleomorphic bacilli [35] that can affect the host immune system through induction of proinflammatory cytokines (e.g., IL-1 $\beta$ and IL-6) via CD4+ $\mathrm{T}$ helper cells and TNF- $\alpha$ [36]. A clinical cross-sectional study found that the presence of $P$. gingivalis and high colonization by $T$. denticola and $P$. intermedia play an important role in severe periodontitis in a Thai population [37]. Besides, the severity of periodontitis in the youths was related to the high level of hormones. In a cohort study of 106 women (50-58 years old), hormone replacement therapy resulted in fewer positive samples for the periodontal pathogens $P$. gingivalis, $P$. intermedia, and Tannerella forsythia in the subgingival plaque [38].

Our results show that Neisseria and Capnocytophaga are increased in men with severe periodontitis, which is consistent with previous results. Minty et al. reported that Capnocytophaga were overrepresented in salivary samples from males compared to females [39]. Capnocytophaga canimorsus is a gram-negative bacillus with unique virulence factors that enable it to evade the human immune system; it is present in the oral cavities of $22 \%$ to $74 \%$ of healthy dogs [40]. Leptotrichia, Prevotella_7, and Prevotella_2 showed no significant difference between males and females. Furthermore, no difference was found between males and females regarding the Simpson and Shannon indices, indicating a large number of similar strains in males and females, which is consistent with the results reported by Chen et al. [41]. In contrast to our results, when Belstrøm et al. used microarrays to examine the oral microbiomes of 292 Danish individuals with low levels of dental caries and periodontitis, they found that diet, BMI, age, and sex did not significantly affect microbial abundance, though socioeconomic status affected the oral microbiome profiles [42]. Other possible factors could explain the differences observed in our study, including genetic variations, social factors, chronology of tooth eruption, and hormonal differences, which could affect the composition of the plaque microbiomes $[14,43]$.

In our study, KEGG analysis found that the immune system, endocrine system, metabolic disease, and infectious disease pathways were significantly changes in women. This may be related to the fact that the female dominant bacteria F. nucleatum had the ability to form a biofilm and coadhere, which can lead to systemic diseases such as urinary tract infection, bacteremia, pericarditis, and otitis media [44, 45]. But the specific mechanism needs to be further studied. This suggests that the imbalance in oral microbiota participates in the regulation of immunological and metabolic homeostasis according to gender differences. However, through the LEfSe analysis, the number of Papillibacter was significantly higher in males. Some studies have found that the high relative abundance of Papillibacter rumminococcacea is related to melanoma patients with a high response to PD-1 immunotherapy [46], indicating that Papillibacter is closely related to immune disease, which is consistent with the enrichment of KEGG analysis in males. Furthermore, Papillibacter can cause local inflammation, resulting in aggregation of $\alpha$-synuclein and generation of Lewy bodies that can participate in the occurrence and development of Parkinson's disease in males [47]. This is possibly related to a phenomenon referred to as the mobile microbiome, which is thought to contribute to systemic disease onset and progression. The phenomenon comprises shifts in the oral microbiome; resulting alterations in the local host-immune response and spillage of proinflammatory mediators into the systemic circulation could influence systemic inflammation and immune system diseases [48]. Moreover, nearly all immune cells express the hormone receptors $[49,50]$, and many immune-related genes possess androgen receptor-responsive elements and estrogen receptor-responsive elements in their promoters, which may be another factor affecting sex differences in the immune responses [51, 52].

\section{Conclusions}

In summary, our study proved the hypothesis of a sexspecific association between the oral microbiomes and severe periodontitis in the youth through $16 \mathrm{~S}$ rRNA gene sequencing. In addition, KEGG pathway analysis predicted that the variation in the oral microbiome may be related to immune homeostasis. Therefore, further investigation is needed to deepen our understanding of the mechanism.

\section{Data Availability}

Raw reads have been deposited at NCBI under the BioProject accession number PRJNA763727.

\section{Conflicts of Interest}

The authors declare that there are no conflicts of interest regarding the publication of this paper. 


\section{Authors' Contributions}

Ya-Qiong Zhao and Ying-Hui Zhou contributed equally to this work.

\section{Acknowledgments}

We thank all of the subgingival plaque donors who participated in our study. This study was supported by the National Natural Science Foundation of China (Grants 81773339,81800788 , and 82001004), the Science and Technology Department of Hunan Province, China (Grants 2017WK2041 and 2018SK52511), the National Natural Science Foundation of Hunan Province (Grant 2018JJ3708), the fund for Xiangya Clinical Medicine Database of Central South University (Grant 2014-ZDYZ-1-16), and the Open Sharing Fund for the Large-scale Instruments and Equipment of Central South University.

\section{References}

[1] M. Freire, K. E. Nelson, and A. Edlund, "The oral hostmicrobial interactome: an ecological chronometer of health?," Trends in Microbiology, vol. 29, no. 6, pp. 551-561, 2021.

[2] J. E. Frencken et al., "Global epidemiology of dental caries and severe periodontitis - a comprehensive review," Journal of Clinical Periodontology, vol. 44, Suppl 18, pp. S94-s105, 2017.

[3] M. A. Peres, L. M. D. Macpherson, R. J. Weyant et al., "Oral diseases: a global public health challenge," Lancet, vol. 394, no. 10194, pp. 249-260, 2019.

[4] J. Jiao, W. Jing, Y. Si et al., "The prevalence and severity of periodontal disease in mainland China: data from the Fourth National Oral Health Survey (2015-2016)," Journal of Clinical Periodontology, vol. 48, no. 2, pp. 168-179, 2021.

[5] L. Abusleme, A. K. Dupuy, N. Dutzan et al., "The subgingival microbiome in health and periodontitis and its relationship with community biomass and inflammation," The ISME Journal, vol. 7, no. 5, pp. 1016-1025, 2013.

[6] M. Bravo-Lopez, V. Villa-Islas, C. Rocha Arriaga et al., "Paleogenomic insights into the red complex bacteriaTannerella forsythiain pre-Hispanic and colonial individuals from Mexico," Philosophical Transactions of the Royal Society of London. Series B, Biological Sciences, vol. 375, no. 1812, p. 20190580, 2020.

[7] G. Hajishengallis, "Immunomicrobial pathogenesis of periodontitis: keystones, pathobionts, and host response," Trends in Immunology, vol. 35, no. 1, pp. 3-11, 2014.

[8] G. Hajishengallis and J. D. Lambris, "Microbial manipulation of receptor crosstalk in innate immunity," Nature Reviews. Immunology, vol. 11, no. 3, pp. 187-200, 2011.

[9] G. Hajishengallis, S. Liang, M. A. Payne et al., "Low-abundance biofilm species orchestrates inflammatory periodontal disease through the commensal microbiota and complement," Cell Host \& Microbe, vol. 10, no. 5, pp. 497-506, 2011.

[10] P. D. Bittner-Eddy, L. A. Fischer, and M. Costalonga, "Identification of gingipain-specific I-A(b) -restricted CD4+ T cells following mucosal colonization with Porphyromonas gingivalis in C57BL/6 mice," Molecular Oral Microbiology, vol. 28, no. 6, pp. 452-466, 2013.

[11] S. L. F. Miranda, J. T. Damasceno, M. Faveri et al., "Brazilian red propolis reduces orange-complex periodontopathogens growing in multispecies biofilms," Biofouling, vol. 35, no. 3, pp. 308-319, 2019.

[12] B. Signat, C. Roques, P. Poulet, and D. Duffaut, "Fusobacterium nucleatum in periodontal health and disease," Current Issues in Molecular Biology, vol. 13, no. 2, pp. 25-36, 2011.

[13] A. Renson, H. E. Jones, F. Beghini et al., "Sociodemographic variation in the oral microbiome," Ann Epidemiol, vol. 35, pp. 73-80.e2, 2019.

[14] P. S. Kumar, "Sex and the subgingival microbiome: do female sex steroids affect periodontal bacteria?," Periodontol 2000, vol. 61, no. 1, pp. 103-124, 2013.

[15] V. Grover, A. Jain, A. Kapoor, R. Malhotra, and G. Singh Chahal, "The gender bender effect in periodontal immune response," Endocrine, Metabolic \& Immune Disorders Drug Targets, vol. 16, no. 1, pp. 12-20, 2016.

[16] M. Umeda, C. Chen, I. Bakker, A. Contreras, J. L. Morrison, and J. Slots, "Risk indicators for harboring periodontal pathogens," Journal of Periodontology, vol. 69, no. 10, pp. 11111118, 1998.

[17] M. S. Tonetti, H. Greenwell, and K. S. Kornman, "Staging and grading of periodontitis: framework and proposal of a new classification and case definition," Journal of Clinical Periodontology, vol. 45, Supplement 20, pp. S149-S161, 2018.

[18] V. Machado, E. M. Aguilera, J. Botelho et al., "Association between periodontitis and high blood pressure: results from the study of periodontal health in Almada-Seixal (SoPHiAS)," Journal of Clinical Medicine, vol. 9, no. 5, p. 1585, 2020.

[19] T. Rognes, T. Flouri, B. Nichols, C. Quince, and F. Mahé, "VSEARCH: a versatile open source tool for metagenomics," PeerJ, vol. 4, article e2584, 2016.

[20] Q. Wang, G. M. Garrity, J. M. Tiedje, and J. R. Cole, "Naive Bayesian classifier for rapid assignment of rRNA sequences into the new bacterial taxonomy," Applied and Environmental Microbiology, vol. 73, no. 16, pp. 5261-5267, 2007.

[21] M. G. Langille et al., "Predictive functional profiling of microbial communities using 16S rRNA marker gene sequences," Nature Biotechnology, vol. 31, no. 9, pp. 814-821, 2013.

[22] T. Z. DeSantis, P. Hugenholtz, N. Larsen et al., "Greengenes, a chimera-checked 16S rRNA gene database and workbench compatible with ARB," Applied and Environmental Microbiology, vol. 72, no. 7, pp. 5069-5072, 2006.

[23] B. Pihlstrom, B. S. Michalowicz, and N. W. Johnson, "Periodontal diseases," The Lancet, vol. 366, no. 9499, pp. 1809$1820,2005$.

[24] F. E. Dewhirst, T. Chen, J. Izard et al., "The human oral microbiome," Journal of Bacteriology, vol. 192, no. 19, pp. 50025017, 2010.

[25] M. Costalonga and M. C. Herzberg, "The oral microbiome and the immunobiology of periodontal disease and caries," Immunology Letters, vol. 162, no. 2, pp. 22-38, 2014.

[26] T. Takagi, Y. Naito, R. Inoue et al., "Differences in gut microbiota associated with age, sex, and stool consistency in healthy Japanese subjects," Journal of Gastroenterology, vol. 54, no. 1, pp. 53-63, 2019.

[27] J. J. Zambon, "Periodontal diseases: microbial factors," Annals of Periodontology, vol. 1, no. 1, pp. 879-925, 1996.

[28] A. D. Kostic, E. Chun, L. Robertson et al., "Fusobacterium nucleatum potentiates intestinal tumorigenesis and modulates the tumor-immune microenvironment," Cell Host Microbe, vol. 14, no. 2, pp. 207-215, 2013. 
[29] W. J. Guan, J. J. Yuan, H. M. Li et al., "Proteobacteria community compositions correlate with bronchiectasis severity," The International Journal of Tuberculosis and Lung Disease, vol. 22, no. 9, pp. 1095-1105, 2018.

[30] W. Xu, W. Zhou, H. Wang, and S. Liang, "Roles of Porphyromonas gingivalis and its virulence factors in periodontitis," Advances in Protein Chemistry and Structural Biology, vol. 120, pp. 45-84, 2020.

[31] G. Papadopoulos, E. O. Weinberg, P. Massari et al., "Macrophage-specific TLR2 signaling mediates pathogen-induced TNF-dependent inflammatory oral bone loss," Journal of Immunology, vol. 190, no. 3, pp. 1148-1157, 2013.

[32] E. V. G. FRANDSEN, K. POULSEN, and M. KILIAN, “Confirmation of the species Prevotella intermedia and Prevotella nigrescens," International Journal of Systematic Bacteriology, vol. 45, no. 3, pp. 429-435, 1995.

[33] Y. Masakiyo, A. Yoshida, Y. Shintani, Y. Takahashi, T. Ansai, and T. Takehara, "The identification of genes specific to Prevotella intermedia and Prevotella nigrescens using genomic subtractive hybridization," Anaerobe, vol. 16, no. 3, pp. 265-269, 2010.

[34] S. M. Guan, M. Zhang, J. J. He, and J. Z. Wu, "Mitogen-activated protein kinases and phosphatidylinositol 3-kinase are involved in Prevotella intermedia -induced proinflammatory cytokines expression in human periodontal ligament cells," Biochemical and Biophysical Research Communications, vol. 386, no. 3, pp. 471-476, 2009.

[35] A. Sharma, S. Inagaki, K. Honma, C. Sfintescu, P. J. Baker, and R. T. Evans, "Tannerella forsythia-induced alveolar bone loss in mice involves leucine-rich-repeat BspA protein," Journal of Dental Research, vol. 84, no. 5, pp. 462-467, 2005.

[36] B. Malinowski, A. Węsierska, K. Zalewska et al., "The role of Tannerella forsythia and Porphyromonas gingivalis in pathogenesis of esophageal cancer," Infect Agent Cancer, vol. 14, p. 3, 2019.

[37] K. Torrungruang, S. Jitpakdeebordin, O. Charatkulangkun, and Y. Gleebbua, "Porphyromonas gingivalis, Aggregatibacter actinomycetemcomitans, and Treponema denticola / Prevotella intermedia co-infection are associated with severe periodontitis in a Thai population," PLoS One, vol. 10, no. 8, article e0136646, 2015.

[38] L. Tarkkila, K. Kari, J. Furuholm, A. Tiitinen, and J. H. Meurman, "Periodontal disease-associated micro-organisms in peri-menopausal and post-menopausal women using or not using hormone replacement therapy. A two-year follow-up study," Oral health, vol. 10, no. 1, 2010.

[39] M. Minty, P. Loubières, T. Canceill et al., "Gender-associated differences in oral microbiota and salivary biochemical parameters in response to feeding," Journal of Physiology and Biochemistry, vol. 77, no. 1, pp. 155-166, 2021.

[40] H. Shin, M. Mally, M. Kuhn, C. Paroz, and G. R. Cornelis, "Escape from immune surveillance by Capnocytophaga canimorsus," The Journal of Infectious Diseases, vol. 195, no. 3, pp. 375-386, 2007.

[41] R. Chen, L. Wang, T. Koch et al., "Sex effects in the association between airway microbiome and asthma," Ann Allergy Asthma Immunol, vol. 125, no. 6, pp. 652-657.e3, 2020.

[42] D. Belstrøm, P. Holmstrup, C. H. Nielsen et al., "Bacterial profiles of saliva in relation to diet, lifestyle factors, and socioeconomic status," Journal of Oral Microbiology, vol. 6, no. 1, 2014.
[43] A. Brodzikowska, R. Górska, and J. Kowalski, "Interleukin-1 genotype in periodontitis," Archivum Immunologiae et Therapiae Experimentalis (Warsz), vol. 67, no. 6, pp. 367-373, 2019.

[44] M. Sanz, A. Marco del Castillo, S. Jepsen et al., "Periodontitis and cardiovascular diseases: consensus report," Journal of Clinical Periodontology, vol. 47, no. 3, pp. 268-288, 2020.

[45] P. S. Zilm and A. H. Rogers, "Co-adhesion and biofilm formation by Fusobacterium nucleatum in response to growth $\mathrm{pH}$," Anaerobe, vol. 13, no. 3-4, pp. 146-152, 2007.

[46] V. Gopalakrishnan, C. N. Spencer, L. Nezi et al., "Gut microbiome modulates response to anti-PD-1 immunotherapy in melanoma patients," Science, vol. 359, no. 6371, pp. 97-103, 2018.

[47] V. A. Petrov, I. V. Saltykova, I. A. Zhukova et al., "Analysis of gut microbiota in patients with Parkinson's disease," Bulletin of Experimental Biology and Medicine, vol. 162, no. 6, pp. 734-737, 2017.

[48] Y. W. Han and X. Wang, "Mobile microbiome: oral bacteria in extra-oral infections and inflammation," Journal of Dental Research, vol. 92, no. 6, pp. 485-491, 2013.

[49] M. Pierdominici et al., "Estrogen receptor profiles in human peripheral blood lymphocytes," Immunology Letters, vol. 132, no. 1-2, pp. 79-85, 2010.

[50] A. Mantalaris, N. Panoskaltsis, Y. Sakai et al., "Localization of androgen receptor expression in human bone marrow," The Journal of Pathology, vol. 193, no. 3, pp. 361-366, 2001.

[51] M. F. Hannah, V. B. Bajic, and S. L. Klein, "Sex differences in the recognition of and innate antiviral responses to Seoul virus in Norway rats," Brain, Behavior, and Immunity, vol. 22, no. 4, pp. 503-516, 2008.

[52] S. Kovats, "Estrogen receptors regulate innate immune cells and signaling pathways," Cellular Immunology, vol. 294, no. 2, pp. 63-69, 2015. 